\title{
Robotic-assisted versus open distal pancreatectomy for benign and low-grade malignant pancreatic tumors: a propensity score-matched study
}

\author{
Yuanchi Weng $^{1}$ (D) $\cdot$ Jiabin Jin $^{1} \cdot$ Zhen Huo $^{1} \cdot$ Yusheng Shi ${ }^{1} \cdot$ Yu Jiang ${ }^{1} \cdot$ Xiaxing Deng $^{1} \cdot$ Chenghong Peng $^{1}$. \\ Baiyong Shen ${ }^{1}$
}

Received: 17 January 2020 / Accepted: 13 May 2020 / Published online: 11 August 2020

(C) The Author(s) 2020

\begin{abstract}
Background This study aimed to compare the short-term outcomes of open and robotic-assisted distal pancreatectomy (ODP and RDP) for benign and low-grade malignant tumors.

Methods The patients who underwent RDP and ODP for benign or low-grade malignant pancreatic tumors at our center were included. After PSM at a 1:1 ratio, the perioperative variations in the two cohorts were compared.

Results After 1:1 PSM, 219 cases of RDP and ODP were recorded. The RDP cohort showed advantages in the operative duration [120 (90-150) min vs 175 (130-210) min, $P<0.001]$, estimated blood loss [50 (30-175) $\mathrm{ml} \mathrm{vs} \mathrm{200} \mathrm{(100-300)} \mathrm{ml,}$ $P<0.001]$, spleen preservation rate $(63.5 \%$ vs $26.5 \%, P<0.001)$, infection rate $(4.6 \%$ vs $12.3 \%, P=0.006)$, and gastrointestinal function recovery [3 (2-4) vs. $3(3-5), P=0.019]$. There were no significant differences in postoperative pancreatic fistula, postoperative hemorrhage, and delayed gastric emptying. Multivariate analysis showed that RDP (HR 0.24; 95\% CI 0.16-0.36, $P<0.001$ ), age (HR 1.02; 95\% CI 1.00-1.03, $P=0.033$ ), tumor size (HR 1.28; 95\% CI 1.17-1.40, $P<0.001$ ), pathological inflammatory neoplasm type (HR 5.12; 95\% CI 2.22-11.81, $P<0.001$ ), and estimated blood loss (HR 1.003; 95\% CI 1.001-1.004, $P<0.001$ ) were independent predictors of spleen preservation; RDP (HR 0.27; 95\% CI 0.17-0.43, $P<0.001$ ), age (HR 1.02; 95\% CI 1.00-1.03, $P=0.022)$, elevated CA 19-9 level (HR 2.55; 95\% CI 1.02-6.39, $P=0.046)$, tumor size (HR 1.44; 95\% CI 1.29-1.61, P<0.001), pathological inflammatory neoplasm type (HR 4.48; 95\% CI 1.69-11.85, $P=0.003$ ), and estimated blood loss (HR 1.003; 95\% CI 1.001-1.004, $P<0.001$ ) were independent predictors of spleen preservation with the Kimura technique.

Conclusion RDP has advantages in the operative time, blood loss, spleen preservation, infection rate, and gastrointestinal function recovery over ODP in treating benign and low-grade malignant pancreatic tumors. The robotic-assisted approach was an independent predictor of spleen preservation and use of the Kimura technique.
\end{abstract}

Keywords Robotic-assisted $\cdot$ Distal pancreatectomy $\cdot$ Benign and low-grade malignant tumors $\cdot$ Spleen preservation

Yuanchi Weng, Jiabin Jin, and Zhen Huo have contributed equally to this work.

Electronic supplementary material The online version of this article (https://doi.org/10.1007/s00464-020-07639-9) contains supplementary material, which is available to authorized users.

Xiaxing Deng

kejiadxx@hotmail.com

$\triangle$ Chenghong Peng

chhpeng@yeah.net

Baiyong Shen

shenby@shsmu.edu.cn

Extended author information available on the last page of the article

\section{Background}

Minimally invasive distal pancreatectomy was first applied in 1992 [1] and has become increasingly popular in recent years [2,3]. Robotic-assisted distal pancreatectomy (RDP) and laparoscopic distal pancreatectomy (LDP), as two kinds of minimally invasive surgery for left-side pancreatic tumors, both showed noninferior short-term outcomes and even oncological outcomes in previous studies [4-6], the newest guideline about minimally invasive pancreas resection, also paid noticeable attention on minimally invasive distal pancreatectomy [7]. While a series of studies have shown the advantages of LDP, a comparison between robotic 
and open approaches for distal pancreatectomy, especially for benign and low-grade malignant tumors, is lacking.

Patients with benign and low-grade malignant pancreatic tumor patients are always priority candidates for minimally invasive pancreatic surgery. For benign and low-grade malignant tumors in the pancreatic body/tail, LDP has already been proven to have a better postoperative effect than open distal pancreatectomy (ODP).

The surgical treatment of benign and low-grade malignant tumors in the pancreatic body/tail always has the potential for spleen-preserving distal pancreatectomy (SPDP). SPDP is associated with lower rates of postoperative complications [8-10], such as thrombocytosis, embolism, and infection. Splenic vessel-sacrificing (Warshaw technique (WT)) SPDP and splenic vessel-preserving (Kimura technique (KT)) SPDP are two methods applied in SPDP [11, 12]. Although more technically challenging, the KT has a lower risk of splenic infarction and gastric varices, which could occur with the WT [13-15].

Therefore, we designed this study with two aims: to compare the short-term outcomes of ODP and RDP for benign and low-grade malignant tumors and to analyze the factors affecting the spleen preservation rate and the use of KT SPDP.

\section{Methods}

We conducted a retrospective study from a prospective database. This study was performed according to the STROBE guidelines [16]. The study protocol was approved by the Institutional Review Board, Ruijin Hospital, and the requirement for informed consent was waived due to the observational and retrospective nature of the study.

\section{Patient selection and study design}

Between February 2012 and March 2019, all hospitalized patients with a preoperative diagnosis of a benign or lowgrade malignant tumor of the pancreatic body/tail at the Pancreatic Surgery Department of Ruijin Hospital were included. Preoperative computed tomography (CT) and magnetic resonance imaging (MRI) scans were routinely performed, and endoscopic ultrasonography was performed for cystic tumors or cases with an unclear diagnosis and surgical indication. The preoperative diagnosis and surgical indication were determined by a multidisciplinary team (MDT) to obtain a more precise and reliable preoperative diagnosis. The MDT consisted of the chief surgeon, first assistant, and at least two professors or associate professors of radiology, and SPDP was performed on an intention-totreat basis.
An open approach was suggested in a few cases with contraindications of minimally invasive surgery, such as complicated abdominal operation history, severe cardiopulmonary complications, or very old age. In the remaining cases, the selection of RDP and ODP was based on patient preference and acceptance. RDP and ODP were performed by the same group of surgeons in the Pancreatic Surgery Department of Ruijin Hospital, and the team had previous experience in ODP (> 300 cases). To minimize selection bias, we also excluded the initial 40 cases of RDP (from our first case of RDP in 2010 to February 2012) according to previous studies about the learning curve for RDP $[17,18]$.

Benign and low-grade malignant tumors were defined as pancreatic tumors without oncological side effects treated by SPDP according to the most recent guidelines, including cystic neoplasms, solid pseudopapillary neoplasms, pancreatic neuroendocrine tumors (G1 and G2 without metastasis), inflammatory neoplasms, and other types of pancreatic cancers.

The exclusion criteria included the following: (1) malignant pancreatic tumors, such as pancreatic ductal adenocarcinoma (PDAC), pancreatic adenosquamous carcinoma, pancreatic acinar cell carcinoma, G3 pancreatic neuroendocrine tumors (PNETs), and other pancreatic tumors with malignant biological behavior; and (2) pancreatic neoplasms with suspicion of metastasis, such as G1/G2 PNETs with liver metastasis.

\section{Surgical protocols}

The da Vinci Si Robotic Surgical System (Intuitive Surgical, Sunnyvale, CA, USA) was applied in this study for RDP cases. The procedure for RDP used in this work is similar to that used in a previous article [19] and can be subdivided into the following steps: (1) First, the tumor was exposed, and the possibility of SPDP was evaluated; difficult mobilization of the splenic vessels and high risk of perioperative massive bleeding with the KT were indications for WT SPDP or splenectomy. (2) For KT SPDP, the distal pancreas was mobilized from the splenic vessels, and the branches of splenic vessels were carefully ligated. (3) For the WT SPDP and DP with splenectomy, the pancreas was dissected with the splenic vessels. DP with splenectomy was performed in the following situations: intraoperative signs of splenic ischemia or infarction or a splenic hilum that was technically unable to be transected. (4) The final steps included pathological examination to determine malignant pathological cases that were inappropriate for spleen preservation and to confirm negative margins. In PNETs larger than $2 \mathrm{~cm}$, regional node lymphadenectomy was performed according to the National Comprehensive Cancer Network (NCCN) guidelines. In WT SPDP cases, we reevaluated the blood 
supply of the spleen. A drainage tube was placed at the surgical site.

\section{Definitions}

Perioperative variables were collected from the hospital's electronic records system; baseline characteristics included age, sex, body mass index (BMI, $\mathrm{kg} / \mathrm{m}^{2}$ ), albumin (ALB) level, previous abdominal surgery history, carbohydrate antigen 19-9 (CA 19-9) level, and American Society of Anesthesiologists Physical Status Classification (ASA score) [20]. We defined portal vein (PV)/superior mesenteric vein (SMV) abutment as PV/SMV compression or lumen narrowing. Tumor size was defined as the longest diameter of the primary tumor or the diameter of the largest tumor in multifocal tumor cases. Regarding intraoperative and postoperative variables, docking time was included in the operative duration in the RDP group, and estimated blood loss was evaluated by the aspirated volume and gauze weight. Postoperative pancreatic fistula (POPF) was defined according to the updated definition of the International Study Group of Pancreatic Fistula [21]; grade A POPF was classified as biochemical leak, and grade $\mathrm{B} / \mathrm{C}$ was classified as clinically relevant POPF. Postpancreatectomy hemorrhage (PPH) and delayed gastric emptying (DGE) represented complications defined by the International Study Group of Pancreatic Surgery [22, 23]. Infection represented surgical site infection, as defined by the Centers for Disease Control and Prevention (CDC) [24] and diagnosed by positive surgical site pathogen culture, either from the drainage or puncture ascites during the postoperative hospitalization. The complication grade was determined according to the Clavien-Dindo classification [25]. Postoperative interventions, such as vascular embolization, vascular stent placement, percutaneous drainage and endoscopic therapy, were also included in the reoperation rate. Oral intake represented the postoperative returning to liquid diet without gastrointestinal symptoms, regardless of the liquid volume. The discharge criteria included: semifluid diet and able to maintain required caloric intake; no need for intravenous fluids; return to independent mobility or baseline mobility for those with previous mobility deficits. The length of stay (LOS) was defined as the number of postoperative days from the operation to discharge.

\section{Matching}

To minimize the selection bias caused by different characteristics of the patients and tumors, RDP cases were matched to ODP cases using propensity scores. Propensity scores were based on the baseline variables age, sex, BMI, ALB level, previous abdominal surgery history, ASA physical status, CA 19-9 level, and PV/SMV abutment, together with the variations in tumor size, pathological type and tumor location. Propensity score matching (PSM) was performed at a 1:1 ratio, and a caliper width of 0.05 standard deviation (SD) was specified.

\section{Statistical analysis}

Continuous variables with a normal distribution are presented as the mean and SD and were analyzed using Student's $t$ test or the paired $t$ test. Continuous variables without a normal distribution are presented as the median and interquartile range (IQR), and the Mann-Whitney $U$ test was used for analysis between the two groups. Categorical variables are presented as frequencies or percentages and were analyzed by the chi-squared test, Fisher's exact test or McNemar's test. Univariate and multivariate logistic regression modeling was performed to analyze the spleen preservation rate and use of the KT. Multivariate analysis was performed after univariate analysis, and the results are presented as hazard ratios (HRs) with 95\% confidence intervals (95\% CIs). All statistical tests were two-sided, and $P<0.05$ was considered to indicate a significant difference. Statistical analyses were performed using the statistical package $\mathrm{R}$ (The R Foundation; https://www.r-project.org; version 3.4.3).

\section{Results}

\section{Study group}

A total of 766 patients were discussed by the MDT and regarded as candidates with benign or low-grade malignant pancreatic tumor patients for spleen-preserving surgery, and these patients underwent DP on an intention-to-treat basis performed by the same surgical team. Eighty-seven cases were excluded from the final analyzed database for different reasons: (1) intraoperative cryosection pathological examination findings with a malignant component $(N=29)$; (2) metastatic disease, including all PNET cases with distant metastasis $(N=12)$; (3) intraoperative decision to convert to enucleation (EN) or middle pancreatectomy (MP) $(N=29)$; and (4) history of Whipple or middle pancreatectomy $(N=17)$. The remaining 679 patients were included in the final analysis; 416 underwent RDP, and 263 underwent ODP. The study flowchart is shown in Fig. 1. There were three cases $(0.7 \%)$ of conversion in the RDP group.

\section{Baseline characteristics}

Before matching, the RDP group had a significantly lower age, lower male patient proportion, higher ALB level, lower rate of previous abdominal surgery, and smaller tumor size; significant differences were also found in the ASA physical 
Fig. 1 Patient selection flowchart

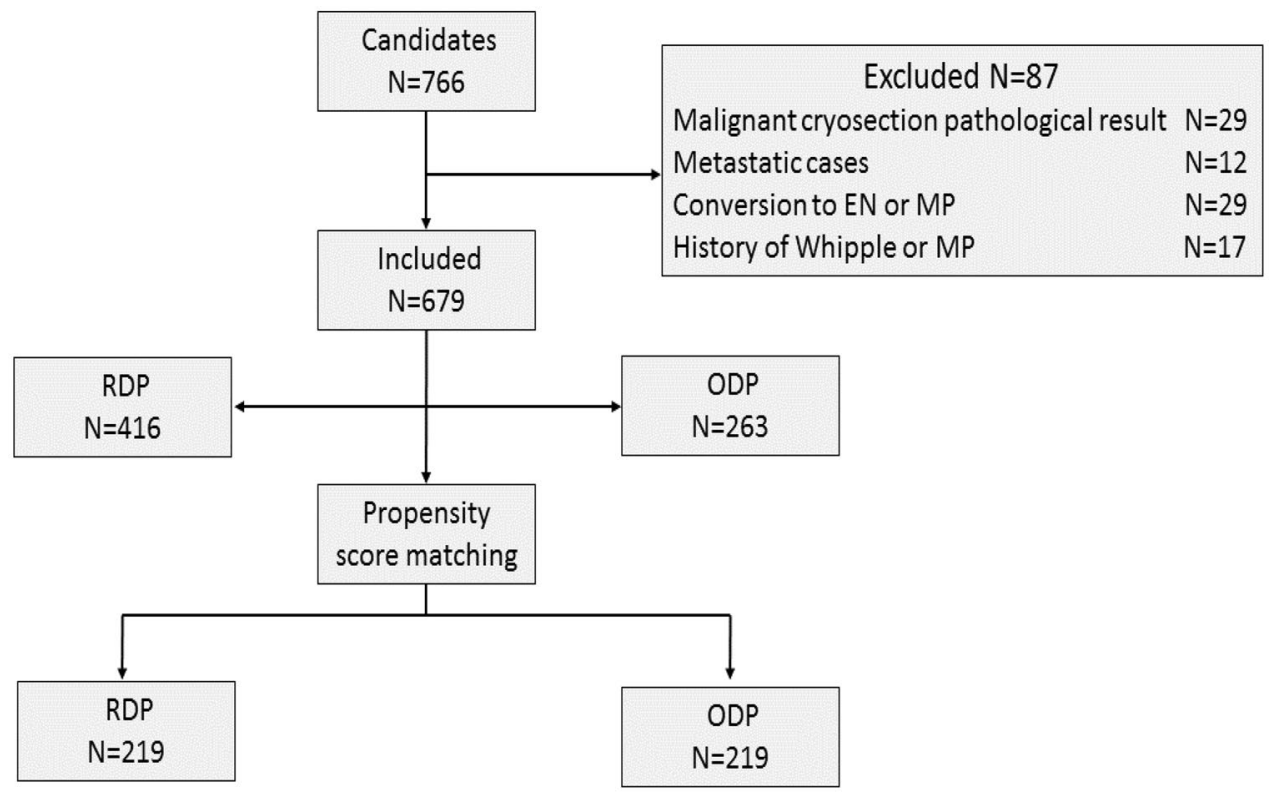

status and pathological type. After PSM at a ratio of $1: 1$, 219 patients were included in each group, and the baseline characteristics became equivalent to minimize selection bias for subsequent analysis (Table 1).

\section{Perioperative outcomes}

There were three cases $(0.7 \%)$ of conversion to laparotomy in the unmatched RDP group. The reasons for conversion in the RDP group included two cases of severe abdominal adhesion and 1 case of uncontrollable intraoperative bleeding from the splenic vein. No vascular resection and reconstruction occurred.

After matching, the RDP cohort had a significantly shorter operative duration (120 min vs. $180 \mathrm{~min}, P<0.001$ ) and less estimated blood loss (50 ml vs. $200 \mathrm{ml}, P<0.001)$ than the ODP cohort. The RDP and ODP cohorts showed similar rates of R0 resection $(95.4 \%$ vs $97.3 \%, P=0.445)$, while the RDP cohort showed a significantly higher spleen preservation rate $(63.5 \%$ vs $26.5 \%, P<0.001)$. In terms of postoperative complications, the two cohorts showed no significant difference in the incidence of POPF, DGE, or PPH, while the RDP cohort showed a lower infection rate $(4.6 \%$ vs $12.3 \%, P=0.006)$ and earlier gastrointestinal function recovery [3 (2-4) vs $3(3-5), P=0.019]$. The number of days to oral intake, severe complications with a Clavien-Dindo score $\geq 3,90$-day mortality rate and postoperative LOS also showed equivalence between the two cohorts (Table 2).

In logistic regression analysis for predicting DP with splenectomy (failure of spleen preservation) and splenic vessel sacrifice (failure of KT SPDP) in DP for benign and low-grade malignant tumors, the robotic approach, together with age, tumor size, pathological type of inflammatory neoplasm, and estimated blood loss, were independent predictors of DP with splenectomy. The robotic approach, together with age, elevated CA 19-9 level, tumor size, pathological type of an inflammatory neoplasm, tumor location in the pancreatic body and neck, and estimated blood loss, were found to be independent predictors of splenic vessel sacrifice (Tables 3, 4).

\section{Discussion}

A minimally invasive approach is considered technically available, safe and feasible for DP in the treatment of leftside pancreatic tumors [5, 26-29]. As an inevitable part of minimally invasive DP, RDP has been frequently analyzed in observational studies, and a series of reports have indicated that RDP results in a shorter operative duration, less blood loss, shorter postoperative hospital stay, higher spleen preservation rate, and comparable morbidity and mortality rates [30-34].

For patients with benign and low-grade malignant pancreatic tumors, good cosmetic effects are required, and low morbidity and organ preservation should be achieved. To compare the perioperative outcomes of RDP and ODP for such benign and low-grade malignant tumors in the pancreatic body/tail, we performed a retrospective analysis using a prospective database. In our study, we collected data from patients with benign and low-grade malignant tumors treated with RDP after the learning curve had been passed for comparison with data from patients treated with ODP during the same time period. PSM has been a popular statistical method in recent years to eliminate bias in observational studies [35], and PSM was applied in our study to eliminate 
Table 1 Demographic and baseline characteristics of the study population

\begin{tabular}{|c|c|c|c|c|c|c|}
\hline & \multicolumn{3}{|c|}{ Before propensity score matching } & \multicolumn{3}{|c|}{ After propensity score matching } \\
\hline & $\operatorname{RDP}(N=416)$ & $\operatorname{ODP}(N=263)$ & $P$ value & $\operatorname{RDP}(N=219)$ & ODP $(N=219)$ & $P$ value \\
\hline Age (years), mean (SD) & $47.8 \pm 15.5$ & $51.6 \pm 15.0$ & 0.002 & $50.4 \pm 15.5$ & $51.0 \pm 14.6$ & 0.651 \\
\hline Sex & & & 0.011 & & & 0.110 \\
\hline Male & $128(30.8 \%)$ & $106(40.3 \%)$ & & $69(31.5 \%)$ & $86(39.3 \%)$ & \\
\hline Female & $288(69.2 \%)$ & $157(59.7 \%)$ & & $150(68.5 \%)$ & $133(60.7 \%)$ & \\
\hline BMI $\left(\mathrm{kg} / \mathrm{m}^{2}\right)$, mean $(\mathrm{SD})$ & $23.2 \pm 3.7$ & $23.1 \pm 3.8$ & 0.784 & $23.2 \pm 3.5$ & $23.2 \pm 3.9$ & 0.817 \\
\hline GLU (mmol/L), median (IQR) & $5.2(4.7-5.7)$ & $5.1(4.6-5.6)$ & 0.662 & $5.3(4.6-5.8)$ & $5.0(4.6-5.6)$ & 0.356 \\
\hline $\operatorname{ALB}(\mathrm{g} / \mathrm{L})$, median (IQR) & $42.0(39.0-45.0)$ & $40.0(37.0-43.0)$ & $<0.001$ & $41.0(38.0-44.0)$ & $40.0(38.0-43.0)$ & 0.199 \\
\hline Abdominal surgery history & $35(8.4 \%)$ & $42(16.0 \%)$ & 0.003 & $27(11.8 \%)$ & $29(13.9 \%)$ & 0.568 \\
\hline ASA score & & & $<0.001$ & & & 0.116 \\
\hline 1 & $330(79.3 \%)$ & $152(57.8 \%)$ & & $157(71.7 \%)$ & $137(62.6 \%)$ & \\
\hline 2 & $76(18.3 \%)$ & $95(36.1 \%)$ & & $53(24.2 \%)$ & $72(32.9 \%)$ & \\
\hline 3 & $10(2.4 \%)$ & $16(6.1 \%)$ & & $9(4.1 \%)$ & $10(4.6 \%)$ & \\
\hline CA $19-9$ & & & 0.053 & & & 0.463 \\
\hline$\leq 35 \mathrm{IU} / \mathrm{L}$ & $385(94.8 \%)$ & $224(90.7 \%)$ & & $205(93.6 \%)$ & $201(91.8 \%)$ & \\
\hline$>35 \mathrm{IU} / \mathrm{L}$ & $21(5.2 \%)$ & $23(9.3 \%)$ & & $14(6.4 \%)$ & $18(8.2 \%)$ & \\
\hline PV/SMV abutment & $7(1.7 \%)$ & $6(2.3 \%)$ & 0.579 & $4(1.8 \%)$ & $5(2.3 \%)$ & 1.000 \\
\hline Tumor size $(\mathrm{cm})$, median (IQR) & $2.8(2.0-4.5)$ & $4.0(2.5-5.6)$ & $<0.001$ & $2.5(2.0-5.0)$ & $3.5(2.2-5.0)$ & 0.154 \\
\hline Pathology & & & 0.010 & & & 0.889 \\
\hline Cystic neoplasm & $192(46.2 \%)$ & $96(36.5 \%)$ & & $88(40.2 \%)$ & $79(36.1 \%)$ & \\
\hline Solid neoplasm (SPT and PNET) & $136(32.7 \%)$ & $82(31.2 \%)$ & & $68(31.1 \%)$ & $74(33.8 \%)$ & \\
\hline IPMN & $40(9.6 \%)$ & $32(12.2 \%)$ & & $24(11.0 \%)$ & $27(12.3 \%)$ & \\
\hline Inflammatory neoplasm & $27(6.5 \%)$ & $34(12.9 \%)$ & & $23(10.5 \%)$ & $25(11.4 \%)$ & \\
\hline Others $^{\mathrm{a}}$ & $21(5.1 \%)$ & $19(7.2 \%)$ & & $16(7.3 \%)$ & $14(6.4 \%)$ & \\
\hline Tumor location & & & 0.169 & & & 0.410 \\
\hline Tail & $172(41.4 \%)$ & $107(40.7 \%)$ & & $98(44.7 \%)$ & $94(42.9 \%)$ & \\
\hline Body-tail junction & $91(21.9 \%)$ & $44(16.7 \%)$ & & $41(18.7 \%)$ & $33(15.1 \%)$ & \\
\hline Body and neck & $153(36.8 \%)$ & $112(42.6 \%)$ & & $80(36.5 \%)$ & $92(42.0 \%)$ & \\
\hline
\end{tabular}

$P$ values $<0.05$ indicate a significant difference between the two groups are given in bold

Others $^{\text {a }}$ including teratoma, mesothelioma, angioma

selection bias caused by baseline population and tumor characteristics, such as age, sex, preoperative ALB level, abdominal surgery history, tumor size, and pathological type. After matching, the equivalence of these baseline indices made the results of the subsequent analysis more robust. A total of 416 cases of RDP and 263 cases of ODP were collected. After 1:1 matching, 219 cases in each group were matched and compared. We found that RDP had advantages in terms of operative duration, estimated blood loss, spleen preservation rate, KT SPDP rate, postoperative surgical site infection rate, and gastrointestinal function recovery. However, postoperative oral intake occurred later in the RDP cohort; this may have been influenced by the different protocols used to determine the postoperative oral intake time between the two cohorts. Altogether, these results reveal that RDP causes less surgical trauma and provides a better foundation for postoperative recovery. The robotic approach has been considered a particularly suitable approach for operations that emphasize meticulous and bloodless dissection, thus potentially expanding the indications for RDP [36, 37]. Studies focusing on RDP and benign and low-grade malignant tumors were separately analyzed, and this series of studies also showed that RDP had noninferior perioperative outcomes when compared with corresponding laparoscopic or open surgery in the treatment of these nonmalignant tumors $[33,38,39]$. These conclusions are in agreement with our results showing that RDP offers acceptable perioperative outcomes for benign and low-grade malignant pancreatic body/tail tumors.

In Guerrini's study, RDP showed advantages over LDP in terms of the conversion rate (8.2 vs. $21.6 \%)$ [40]. In benign cases, a lower conversion rate indicates a better cosmetic effect. In our study, there were 3 cases $(0.7 \%)$ of conversion to laparotomy in the RDP group, and severe abdominal adhesion and uncontrollable intraoperative bleeding led to conversion in all intraductal papillary mucinous neoplasia 
Table 2 Intraoperative and perioperative characteristics of the population before and after matching

\begin{tabular}{|c|c|c|c|c|c|c|}
\hline & \multicolumn{3}{|c|}{ Before propensity score matching } & \multicolumn{3}{|c|}{ After propensity score matching } \\
\hline & $\operatorname{RDP}(N=416)$ & $\operatorname{ODP}(N=263)$ & $P$ value & $\operatorname{RDP}(N=219)$ & $\operatorname{ODP}(N=219)$ & $P$ value \\
\hline Conversion to laparotomy & 3 & l & I & 1 & l & l \\
\hline Operative time (min), median (IQR) & $120(90-150)$ & $180(130-210)$ & $<0.001$ & $120(90-150)$ & $175(130-210)$ & $<0.001$ \\
\hline Estimated blood loss (ml), median (IQR) & $50(30-150)$ & $200(100-400)$ & $<0.001$ & $50(30-175)$ & $200(100-300)$ & $<0.001$ \\
\hline $\mathrm{R} 0$ resection, $n(\%)$ & $402(96.6 \%)$ & $256(97.3 \%)$ & 0.606 & $209(95.4 \%)$ & $213(97.3 \%)$ & 0.445 \\
\hline Spleen preservation, $n(\%)$ & $277(66.6 \%)$ & $65(24.7 \%)$ & $<0.001$ & $139(63.5 \%)$ & $58(26.5 \%)$ & $<0.001$ \\
\hline Spleen preservation method, $n(\%)$ & & & $<0.001$ & & & $<0.001$ \\
\hline Splenectomy & $139(33.4 \%)$ & $198(75.3 \%)$ & & $80(36.5 \%)$ & $161(73.5 \%)$ & \\
\hline KT & $202(48.6 \%)$ & $39(14.8 \%)$ & & $101(46.1 \%)$ & $35(16.0 \%)$ & \\
\hline WT & $75(18.0 \%)$ & $26(9.9 \%)$ & & $38(17.4 \%)$ & $23(10.5 \%)$ & \\
\hline POPF, $n(\%)$ & $93(22.4 \%)$ & $82(31.2 \%)$ & 0.010 & $53(24.2 \%)$ & $70(32.0 \%)$ & 0.071 \\
\hline CR-POPF, $n(\%)$ & $53(12.7 \%)$ & $47(17.9 \%)$ & 0.066 & $32(14.6 \%)$ & $41(18.7 \%)$ & 0.249 \\
\hline DGE, $n(\%)$ & $6(1.4 \%)$ & $5(1.9 \%)$ & 0.758 & $5(2.3 \%)$ & $5(2.3 \%)$ & 1.000 \\
\hline Infection, $n(\%)$ & $20(4.8 \%)$ & $33(12.6 \%)$ & $<0.001$ & $10(4.6 \%)$ & $27(12.3 \%)$ & 0.006 \\
\hline $\mathrm{PPH}, n(\%)$ & $10(2.4 \%)$ & $6(2.3 \%)$ & 0.918 & $8(3.7 \%)$ & $5(2.3 \%)$ & 0.573 \\
\hline Others $^{\mathrm{a}}, n(\%)$ & $10(2.4 \%)$ & $9(3.4 \%)$ & 0.433 & $7(3.2 \%)$ & $6(2.7 \%)$ & 1.000 \\
\hline Reoperation, $n(\%)$ & $11(2.6 \%)$ & $7(2.7 \%)$ & 0.989 & $8(3.7 \%)$ & $7(3.2 \%)$ & 1.000 \\
\hline Clavien-Dindo $\geq 3$ & $12(2.9 \%)$ & $10(3.8 \%)$ & 0.511 & $9(4.1 \%)$ & $8(3.7 \%)$ & 1.000 \\
\hline 90-day mortality, $n(\%)$ & $1(0.2 \%)$ & $2(0.8 \%)$ & 0.563 & $1(0.5 \%)$ & $1(0.5 \%)$ & 1.000 \\
\hline Readmission, $n(\%)$ & $11(2.6 \%)$ & $14(5.3 \%)$ & 0.071 & $7(3.2 \%)$ & $11(5.0 \%)$ & 0.470 \\
\hline GI function, days, median (IQR) & $3(2-4)$ & $4(3-5)$ & $<0.001$ & $3(2-4)$ & $3(3-5)$ & 0.019 \\
\hline Oral intake, days, median (IQR) & $4.5(3-7)$ & $3(2-5)$ & 0.005 & $5(3-7)$ & $3(2-5)$ & 0.006 \\
\hline LOS (days), median (IQR) & $13(11-19)$ & $16(12-21)$ & 0.029 & $14(11-19)$ & $16(11.5-20)$ & 0.407 \\
\hline
\end{tabular}

$P$ values $<0.05$ indicate a significant difference between the two groups are given in bold

$R D P$ robotic-assisted distal pancreatectomy, $O D P$ open distal pancreatectomy, $K T$ Kimura technique, WT Warshaw technique, $P O P F$ postoperative pancreatic fistula, $C R-P O P F$ clinically relevant POPF (ISGPF grade B and C), $P P H$ post pancreatectomy hemorrhage; $D G E$ delayed gastric emptying, LOS postoperative length of stay; others ${ }^{\text {a }}$ : including pneumonia, deep vein thrombosis, heart disease, cerebral hemorrhage, pleural effusion

(IPMN) cases. In all the benign and low-grade malignant tumor cases, the conversion was always caused by IPMN and inflammatory neoplasms, and the precise dissection and suturing of the robotic system consistently provided better management in potential conversion cases. Moreover, the robotic approach always achieved good cosmetic effects.

SPDP, as an important technique in organ-preserving surgery for pancreatic body/tail nonmalignant tumors, has both hematological and immunological advantages, as proven by a series of studies [11, 12, 41, 42]. The WT is a relatively easy spleen-preserving method, and the spleen can often be vascularized by short gastric vessels and the left gastroepiploic artery. The feasibility and acceptable short-term outcomes of WT SPDP have also been proven in different studies [43, 44]. However, WT SPDP is associated with the risk of splenic infarction [45], and there are still some concerns about the long-term outcomes of the WT, such as gastric varices and the risk of gastric bleeding [46]. KT SPDP is the first choice and preferred method for pancreatic body/tail tumors with spleen preservation potential. After
PSM to minimize selection bias, the RDP cohort showed a significantly higher spleen preservation rate and a higher rate of KT SPDP. In the RDP and ODP cohorts, the approaches for pancreatic parenchyma mobilization showed different trends, with a "bottom-up" view in RDP, which was differed from the "top-down" view in ODP. In our clinical practice, the intraoperative bleeding that causes the failure of KT SPDP is often caused by splenic vein bleeding when separating the pancreatic parenchyma from the splenic vessels. Using the robotic surgical system, the 3D view, EndoWrist and tremor elimination all helped to provide more delicate manipulation, precise dissection of the splenic vessels and more meticulous ligation or suturing the small branches of the splenic vessels, which may allow the difficulties in laparoscopic DP with spleen-preserving intention to be better overcome [19]. The advantages of SPDP were reconfirmed by the univariate and multivariate analyses. Age, tumor size, a higher proportion of inflammatory neoplasms and blood loss were independent risk factors of the spleen preservation rate and the KT SPDP rate. Larger tumor size and the 
Table 3 Logistic regression analysis predicting splenectomy (failure of spleen preservation)

\begin{tabular}{|c|c|c|c|c|}
\hline & \multicolumn{2}{|l|}{ Univariate analysis } & \multicolumn{2}{|l|}{ Multivariate analysis } \\
\hline & $\mathrm{HR}(95 \% \mathrm{CI})$ & $P$ value & $\mathrm{HR}(95 \% \mathrm{CI})$ & $P$ value \\
\hline \multicolumn{5}{|l|}{ Approach } \\
\hline ODP & Ref & & & \\
\hline RDP & $0.165(0.117,0.233)$ & $<0.001$ & $0.239(0.158,0.361)$ & $<0.001$ \\
\hline Age & $1.014(1.004,1.024)$ & 0.008 & $1.015(1.001,1.029)$ & $\mathbf{0 . 0 3 3}$ \\
\hline \multicolumn{5}{|l|}{ Sex } \\
\hline Male & Ref & & & \\
\hline Female & $0.696(0.506,0.956)$ & 0.025 & $0.823(0.537,1.260)$ & 0.370 \\
\hline BMI & $0.984(0.945,1.024)$ & 0.428 & & \\
\hline ALB & $0.956(0.926,0.988)$ & 0.007 & $0.990(0.948,1.034)$ & 0.647 \\
\hline Abdominal surgery history & $1.047(0.651,1.683)$ & 0.850 & & \\
\hline \multicolumn{5}{|l|}{ ASA score } \\
\hline 1 & Ref & & & \\
\hline 2 & $1.072(0.756,1.520)$ & 0.695 & & \\
\hline 3 & $2.002(0.875,4.579)$ & 0.100 & & \\
\hline \multicolumn{5}{|l|}{ CA 19-9 (IU/L) } \\
\hline$\leq 35$ & Ref & & & \\
\hline$>35$ & $2.296(1.194,4.416)$ & 0.013 & $1.696(0.785,3.666)$ & 0.179 \\
\hline PV/SMV abutment & $3.456(0.943,12.669)$ & 0.061 & & \\
\hline Tumor size & $1.283(1.192,1.381)$ & $<0.001$ & $1.283(1.173,1.404)$ & $<0.001$ \\
\hline \multicolumn{5}{|l|}{ Pathology } \\
\hline Cystic neoplasm & Ref & & & \\
\hline Solid neoplasm (SPT and PNET) & $1.312(0.921,1.868)$ & 0.133 & $1.634(1.043,2.560)$ & 0.032 \\
\hline IPMN & $1.608(0.957,2.703)$ & 0.073 & $1.565(0.827,2.962)$ & 0.169 \\
\hline Inflammatory neoplasm & $6.939(3.388,14.214)$ & $<0.001$ & $5.121(2.220,11.811)$ & $<0.001$ \\
\hline Others & $1.113(0.572,2.165)$ & 0.752 & $0.884(0.386,2.027)$ & 0.771 \\
\hline \multicolumn{5}{|l|}{ Tumor location } \\
\hline Tail & Ref & & & \\
\hline Body-tail junction & $1.051(0.697,1.585)$ & 0.813 & & \\
\hline Body and neck & $1.108(0.791,1.551)$ & 0.551 & & \\
\hline Operative time & $1.008(1.005,1.011)$ & $<0.001$ & $0.998(0.994,1.002)$ & 0.245 \\
\hline Estimated blood loss & $1.004(1.003,1.005)$ & $<0.001$ & $1.003(1.001,1.004)$ & $<0.001$ \\
\hline
\end{tabular}

$P$ values $<0.05$ indicate a significant difference between the two groups are given in bold nature of the inflammatory neoplasms were often associated with more difficult manipulation of the splenic vessels, and blood loss was often associated with surgical trauma and reflected the difficulty of the procedures. An elevated CA 19-9 level was identified in 44 cases in our study and was found to be an independent predictor of splenic vessel sacrifice (WT SPDP and DP with splenectomy). Elevated CA 19-9 levels have been reported to be associated with acute or chronic pancreatitis even in benign pancreatic tumors [47]. The inflammation caused by some types of benign and lowgrade malignant tumors, such as inflammatory neoplasms and IPMN, may increase the level of CA 19-9 and ultimately cause the failure of splenic vessel preservation, which may explain why an elevated CA 19-9 level was an independent risk factor for KT SPDP.
The low morbidity rate, especially the lower rate of severe complications (Clavien-Dindo score $\geq 3$ ), is also crucial in benign and low-grade malignant pancreatic tumors. POPF, as the most common postoperative complication, may cause subsequent intraoperative infection and $\mathrm{PPH}$. The overall POPF rate was $24.2 \%$ in the RDP group vs $32.0 \%$ in the ODP group, and the corresponding clinically relevant POPF rates (grade $\mathrm{B}$ and $\mathrm{C}$ ) were $14.6 \%$ vs $18.7 \%$, respectively, both without a significant difference. In our study, we transected the pancreas with a stapler in most cases; thus, we did not need to address the pancreatic stump. In other cases in which we transected the pancreas with a harmonic scalpel, we always found the pancreatic duct and ligated it with sutures; the robotic system provided an enlarged view and allowed delicate manipulation, which facilitated handling of the pancreatic stump and reduced the POPF rate. The 
Table 4 Logistic regression analysis predicting Warshaw SPDP and splenectomy (failure of Kimura SPDP)

\begin{tabular}{|c|c|c|c|c|}
\hline & \multicolumn{2}{|l|}{ Univariate analysis } & \multicolumn{2}{|l|}{ Multivariate analysis } \\
\hline & HR $(95 \% \mathrm{CI})$ & $P$ value & HR $(95 \%$ CI $)$ & $P$ value \\
\hline \multicolumn{5}{|l|}{ Approach } \\
\hline ODP & Ref & & & \\
\hline RDP & $0.184(0.125,0.273)$ & $<0.001$ & $0.265(0.165,0.425)$ & $<0.001$ \\
\hline Age & $1.011(1.000,1.021)$ & 0.044 & $1.017(1.002,1.031)$ & 0.022 \\
\hline \multicolumn{5}{|l|}{ Sex } \\
\hline Male & Ref & & & \\
\hline Female & $0.840(0.602,1.173)$ & 0.307 & & \\
\hline BMI & $0.968(0.928,1.009)$ & 0.125 & & \\
\hline ALB & $0.980(0.948,1.012)$ & 0.223 & & \\
\hline Abdominal surgery history & $1.090(0.660,1.798)$ & 0.737 & & \\
\hline \multicolumn{5}{|l|}{ ASA score } \\
\hline 1 & Ref & & & \\
\hline 2 & $1.064(0.739,1.532)$ & 0.739 & & \\
\hline 3 & $2.416(0.895,6.519)$ & 0.082 & & \\
\hline \multicolumn{5}{|l|}{ CA 19-9 (IU/L) } \\
\hline$\leq 35$ & Ref & & & \\
\hline$>35$ & $3.054(1.339,6.965)$ & 0.008 & $2.548(1.015,6.393)$ & 0.046 \\
\hline PV/SMV abutment & $3,265,018.587(0.000$, Inf $)$ & 0.970 & & \\
\hline Tumor size & $1.439(1.308,1.584)$ & $<0.001$ & $1.443(1.289,1.614)$ & $<0.001$ \\
\hline \multicolumn{5}{|l|}{ Pathology } \\
\hline Cystic neoplasm & Ref & & & \\
\hline Solid neoplasm (SPT and PNET) & $0.921(0.642,1.322)$ & 0.655 & $1.268(0.812,1.982)$ & 0.297 \\
\hline IPMN & $1.200(0.696,2.070)$ & 0.512 & $1.303(0.681,2.492)$ & 0.424 \\
\hline Inflammatory neoplasm & $5.500(2.291,13.203)$ & $<0.001$ & $4.477(1.692,11.845)$ & 0.003 \\
\hline Others & $0.812(0.415,1.588)$ & 0.542 & $0.913(0.402,2.074)$ & 0.827 \\
\hline \multicolumn{5}{|l|}{ Tumor location } \\
\hline Tail & Ref & & & \\
\hline Body-tail junction & $1.237(0.807,1.897)$ & 0.329 & $1.487(0.886,2.494)$ & 0.133 \\
\hline Body and neck & $1.449(1.018,2.063)$ & 0.040 & $1.692(1.099,2.607)$ & 0.017 \\
\hline Operative time & $1.008(1.005,1.011)$ & $<0.001$ & $0.999(0.995,1.003)$ & 0.554 \\
\hline Estimated blood loss & $1.004(1.003,1.006)$ & $<0.001$ & $1.003(1.001,1.004)$ & 0.001 \\
\hline
\end{tabular}

$P$ values $<0.05$ indicate a significant difference between the two groups are given in bold
POPF rate in our study is comparable to that in other series of RDP, such as the rate of $17 \%$ reported by Gavriilidis in a systematic review and network meta-analysis [48]. PPH, which is a severe complication after DP, always requires reoperation (including intervention). In our initial practice, before we had mastered this approach, massive PPH with hypovolemic shock occurred in three cases of RDP with the $\mathrm{KT}$, and reoperation was performed (including digital subtraction angiography, celiac artery angiography and splenic artery embolization). After passing the learning curve, we performed more precise manipulations of the small branches of the pancreatic transverse and dorsal arteries, and massive PPH seldom occurred.

There were several limitations to this study, which should be noted. Although the PSM method was applied to reduce selection bias, the retrospective nature of the study cannot be ignored. In addition, despite preoperative MDT discussion and performing the operations based on the intentionto-treat principle, some subjective factors still influenced the intraoperative decision, such as inflammation, adhesions and difficulty in mobilizing the splenic vessels, which may influence the surgeon's choice of the KT, the WT or splenectomy. Third, the follow-up data were missing in some cases, which prevented further analysis of the long-term outcomes between the RDP and ODP groups. Finally, as an irreplaceable minimally invasive pancreatic surgery, LDP also plays an important role in patient treatment, but in our department, since the introduction of the da Vinci robotic system in 2010, many LDP surgeries for benign and low-grade malignant tumors have been replaced by RDP according to our initial experience and that of other surgeons [31, 45]. Therefore, a lack of sufficient clinical data for LDP prevents 
subsequent comparisons among robotic, laparoscopic and open approaches for DP.

\section{Conclusions}

For benign and low-grade malignant tumors in the pancreatic body/tail, the perioperative outcomes of RDP were better than those of ODP in terms of the operative duration, estimated blood loss, spleen preservation, infection rate and gastrointestinal function recovery. The robotic approach was an independent predictor of both spleen preservation and KT SPDP. However, this was a retrospective study with inherent drawbacks. Although we used the PSM method to minimize bias, further randomized controlled studies should be designed to verify the value of RDP for benign and lowgrade malignant tumors.

\section{Compliance with ethical standards}

Disclosures Yuanchi Weng, Jiabin Jin, Zhen Huo, Yusheng Shi, Yu Jiang, Xiaxing Deng, Chenghong Peng, and Baiyong Shen declare that they have no conflicts of interest or financial ties to disclose.

Open Access This article is licensed under a Creative Commons Attribution 4.0 International License, which permits use, sharing, adaptation, distribution and reproduction in any medium or format, as long as you give appropriate credit to the original author(s) and the source, provide a link to the Creative Commons licence, and indicate if changes were made. The images or other third party material in this article are included in the article's Creative Commons licence, unless indicated otherwise in a credit line to the material. If material is not included in the article's Creative Commons licence and your intended use is not permitted by statutory regulation or exceeds the permitted use, you will need to obtain permission directly from the copyright holder. To view a copy of this licence, visit http://creativecommons.org/licenses/by/4.0/.

\section{References}

1. Gagner M, Pomp A, Herrera MF (1996) Early experience with laparoscopic resections of islet cell tumors. Surgery 120:1051-1054

2. Lyman WB, Passeri M, Sastry A et al (2019) Robotic-assisted versus laparoscopic left pancreatectomy at a high-volume, minimally invasive center. Surg Endosc 33:2991-3000

3. Magge DR, Zenati MS, Hamad A et al (2018) Comprehensive comparative analysis of cost-effectiveness and perioperative outcomes between open, laparoscopic, and robotic distal pancreatectomy. HPB 20:1172-1180

4. Yang DJ, Xiong JJ, Lu HM et al (2019) The oncological safety in minimally invasive versus open distal pancreatectomy for pancreatic ductal adenocarcinoma: a systematic review and metaanalysis. Sci Rep 9:1159

5. Hong S, Song KB, Madkhali AA et al (2019) Robotic versus laparoscopic distal pancreatectomy for left-sided pancreatic tumors: a single surgeon's experience of 228 consecutive cases. Surg Endosc. https://doi.org/10.1007/s00464-019-07047-8
6. Qu L, Zhiming Z, Xianglong T et al (2018) Short- and mid-term outcomes of robotic versus laparoscopic distal pancreatosplenectomy for pancreatic ductal adenocarcinoma: a retrospective propensity score-matched study. Int J Surg 55:81-86

7. Asbun HJ, Moekotte AL, Vissers FL et al (2020) The Miami international evidence-based guidelines on minimally invasive pancreas resection. Ann Surg 271:1-14

8. Shi N, Liu SL, Li YT et al (2016) Splenic preservation versus splenectomy during distal pancreatectomy: a systematic review and meta-analysis. Ann Surg Oncol 23:365-374

9. Pendola F, Gadde R, Ripat C et al (2017) Distal pancreatectomy for benign and low grade malignant tumors: short-term postoperative outcomes of spleen preservation-a systematic review and update meta-analysis. J Surg Oncol 115:137-143

10. He Z, Qian D, Hua J et al (2014) Clinical comparison of distal pancreatectomy with or without splenectomy: a meta-analysis. PLoS ONE 9:e91593

11. Warshaw AL (1988) Conservation of the spleen with distal pancreatectomy. Arch Surg 123:550-553

12. Kimura W, Inoue $\mathrm{T}$, Futakawa $\mathrm{N}$ et al (1996) Spleen-preserving distal pancreatectomy with conservation of the splenic artery and vein. Surgery 120:885-890

13. Miura F, Takada T, Asano T et al (2005) Hemodynamic changes of splenogastric circulation after spleenpreserving pancreatectomy with excision of splenic artery and vein. Surgery 138:518-522

14. Sarin SK, Lahoti D, Saxena SP et al (1992) Prevalence, classification and natural history of gastric varices: a long-term follow-up study in 568 portal hypertension patients. Hepatology 16:1343-1349

15. Balzano G, Zerbi A, Di Carlo V (2007) Spleen-preserving distal pancreatectomy with excision of splenic artery and vein: a cautionary note. World J Surg 31:1530

16. von Elm E, Altman DG, Egger M et al (2007) The strengthening the reporting of observational studies in epidemiology (STROBE) statement: guidelines for reporting observational studies. Lancet 370:1453-1457

17. Shakir M, Boone BA, Polanco PM et al (2015) The learning curve for robotic distal pancreatectomy: an analysis of outcomes of the first 100 consecutive cases at a high-volume pancreatic centre. HPB 17:580-586

18. Napoli N, Kauffmann EF, Perrone VG et al (2015) The learning curve in robotic distal pancreatectomy. Updates Surg 67:257-264

19. Chen S, Zhan Q, Chen JZ et al (2015) Robotic approach improves spleen-preserving rate and shortens postoperative hospital stay of laparoscopic distal pancreatectomy: a matched cohort study. Surg Endosc 29:3507-3518

20. Kuza CM, Hatzakis G, Nahmias JT (2017) The assignment of American Society of Anesthesiologists physical status classification for adult polytrauma patients: results from a survey and future considerations. Anesth Analg 125:1960-1966

21. Bassi C, Marchegiani G, Dervenis C et al (2017) The 2016 update of the International Study Group (ISGPS) definition and grading of postoperative pancreatic fistula: 11 years after. Surgery 161:584-591

22. Wente MN, Veit JA, Bassi C et al (2007) Postpancreatectomy hemorrhage (PPH): an International Study Group of Pancreatic Surgery (ISGPS) definition. Surgery 142:20-25

23. Wente MN, Bassi C, Dervenis C et al (2007) Delayed gastric emptying (DGE) after pancreatic surgery: a suggested definition by the International Study Group of Pancreatic Surgery (ISGPS). Surgery 142:761-768

24. Sawyer RG, Claridge JA, Nathens AB et al (2015) Trial of shortcourse antimicrobial therapy for intraabdominal infection. $\mathrm{N}$ Engl J Med 372:1996-2005 
25. Dindo D, Demartines N, Clavien PA (2004) Classification of surgical complications: a new proposal with evaluation in a cohort of 6336 patients and results of a survey. Ann Surg 240:205-213

26. Sánchez-Cabús S, Adam JP, Pittau G et al (2016) Laparoscopic left pancreatectomy: early results after 115 consecutive patients. Surg Endosc 30:4480-4488

27. Jean-Philippe A, Alexandre J, Christophe L et al (2013) Laparoscopic spleen-preserving distal pancreatectomy: splenic vessel preservation compared with the Warshaw technique. JAMA Surg 148:246-252

28. de Rooij T, Klompmaker S, Abu Hilal M et al (2016) Laparoscopic pancreatic surgery for benign and malignant disease. Nat Rev Gastroenterol Hepatol 13:227-238

29. Alfieri S, Boggi U, Butturini G et al (2020) Full robotic distal pancreatectomy: safety and feasibility analysis of a multicenter cohort of 236 patients. Surg Innov. https://doi.org/10.1177/15533 50619868112

30. Huang B, Feng L, Zhao J (2016) Systematic review and metaanalysis of robotic versus laparoscopic distal pancreatectomy for benign and malignant pancreatic lesions. Surg Endosc 30:4078-4085

31. Gavriilidis P, Lim C, Menahem B et al (2016) Robotic versus laparoscopic distal pancreatectomy - the first meta-analysis. HPB 18:567-574

32. Zhou JY, Xin C, Mou YP et al (2016) Robotic versus laparoscopic distal pancreatectomy: a meta-analysis of short-term outcomes. PLoS ONE 11:e0151189

33. Daouadi M, Zureikat AH, Zenati MS et al (2013) Robot-assisted minimally invasive distal pancreatectomy is superior to the laparoscopic technique. Ann Surg 257:128-132

34. Butturini G, Damoli I, Crepaz L et al (2015) A prospective nonrandomised single-center study comparing laparoscopic and robotic distal pancreatectomy. Surg Endosc 29:3163-3170

35. Austin PC (2011) Comparing paired vs non-paired statistical methods of analyses when making inferences about absolute risk reductions in propensity-score matched samples. Stat Med 30:1292-1301

36. Marino MV, Shabat G, Gulotta G et al (2018) From illusion to reality: a brief history of robotic surgery. Surg Innov 25:291-296

37. Giulianotti PC, Sbrana F, Bianco FM et al (2010) Robot-assisted laparoscopic pancreatic surgery: single-surgeon experience. Surg Endosc 24:1646-1657
38. Kang CM, Kim DH, Lee WJ et al (2011) Conventional laparoscopic and robot-assisted spleen-preserving pancreatectomy: does da Vinci have clinical advantages? Surg Endosc 25:2004-2009

39. Ito M, Asano Y, Shimizu T et al (2014) Comparison of standard laparoscopic distal pancreatectomy with minimally invasive distal pancreatectomy using the da Vinci S system. Hepatogastroenterology 61:493-496

40. Guerrini GP, Lauretta A, Belluco C et al (2017) Robotic versus laparoscopic distal pancreatectomy: an up-to-date meta-analysis. BMC Surg 17:105

41. Shoup M, Brennan MF, McWhite K et al (2002) The value of splenic preservation with distal pancreatectomy. Arch Surg 137:164-168

42. Benoist S, Dugué L, Sauvanet A et al (1999) Is there a role of preservation of the spleen in distal pancreatectomy? J Am Coll Surg 188:255-260

43. Butturini G, Inama M, Malleo G et al (2012) Perioperative and long-term results of laparoscopic spleen-preserving distal pancreatectomy with or without splenic vessels conservation: a retrospective analysis. J Surg Oncol 105:387-392

44. Worhunsky DJ, Zak Y, Dua MM et al (2014) Laparoscopic spleenpreserving distal pancreatectomy: the technique must suit the lesion. J Gastrointest Surg 18:1445-1451

45. Eckhardt S, Schicker C, Maurer E et al (2016) Robotic-assisted approach improves vessel preservation in spleen-preserving distal pancreatectomy. Dig Surg 33:406-413

46. Miura F, Takada T, Asano T et al (2005) Hemodynamic changes of splenogastric circulation after spleen-preserving pancreatectomy with excision of splenic artery and vein. Surgery 138:518-522

47. Binicier OB, Pakoz ZB (2019) CA 19-9 levels in patients with acute pancreatitis due to gallstone and metabolic/toxic reasons. Rev Assoc Med Bras 65:965-970

48. Gavriilidis P, Roberts KJ, Sutcliffe RP (2019) Comparison of robotic vs laparoscopic vs open distal pancreatectomy. A systematic review and network meta-analysis. HPB 21:1268-1276

Publisher's Note Springer Nature remains neutral with regard to jurisdictional claims in published maps and institutional affiliations.

\section{Affiliations}

\section{Yuanchi Weng ${ }^{1} \mathbb{D} \cdot$ Jiabin Jin $^{1} \cdot$ Zhen Huo $^{1} \cdot$ Yusheng Shi ${ }^{1} \cdot$ Yu Jiang ${ }^{1} \cdot$ Xiaxing Deng $^{1} \cdot$ Chenghong Peng $^{1}$. Baiyong Shen ${ }^{1}$}

1 Department of General Surgery, Pancreatic Disease Center, Ruijin Hospital, Shanghai Jiao Tong University School of Medicine, No. 197 Ruijin Er Road, Huangpu District, Shanghai 200025, China 